\title{
Correlation of Kynurenine Excretion with Liver Tryptophan Pyrrolase Levels in Disease and after Hydrocortisone Induction *
}

\author{
Kurt Altman † and Olga Greengard \\ (From the Department of Internal Medicine, New York Medical College, and the Institute for \\ Muscle Disease, Inc., New York, N. Y., and the Department of Biological Chemistry, \\ Harvard Medical School, and Cancer Research Institute, New England \\ Deaconess Hospital, Boston, Mass.)
}

In a large variety of apparently unrelated diseases, the major pathway of tryptophan metabolism, that which proceeds through kynurenine, has been observed to be abnormal. The deviation is a quantitative one: the urinary excretion of one or more normal metabolites is high. Attempts to explain this phenomenon have largely been restricted to hypotheses that assume a "metabolic block" in the catabolism of these metabolites, as exemplified by pyridoxine deficiency. However, it seems unlikely that the variety of conditions associated with the increased excretion of these tryptophan metabolites, e.g., Hodgkin's disease, rheumatoid arthritis, schizophrenia, porphyria, renal tuberculosis, and aplastic anemia, to mention only a few that are included in a recent review (1), would all be coupled specifically with pyridoxine deficiency. Furthermore, the term "metabolic block" is of doubtful meaning in conditions not involving the inborn absence of any one enzyme. The accumulation of an intermediary metabolite like kynurenine must be the result of an enzymic imbalance (2): a relative increase in its rate of formation or a relative decrease in its rate of degradation (of which absence of an enzyme is the extreme case). The present study is chiefly concerned with the former possibility.

The oxidation of tryptophan is catalyzed by tryptophan pyrrolase, which was first characterized in mammalian liver in 1950 by Knox and Mehler

\footnotetext{
* Submitted for publication April 4, 1966; accepted June 16, 1966.

This work was supported by the Arthritis Foundation and by U. S. Public Health Service grant CA 08676-01 from the National Cancer Institute.

$\dagger$ Address requests for reprints to Dr. Kurt Altman, New York Medical College, Flower and Fifth Ave. Hospitals, Fifth Ave. at 106th St., New York, N. Y. 10029.
}

(3). It is the first enzyme on the kynurenine pathway and so determines how much tryptophan enters the pathway. The regulatory role of tryptophan pyrrolase is also indicated by the fact that its level in rat liver varies significantly and appropriately under different physiological conditions (4). The observed rises in activity under such conditions are called inductions because they reflect increases in the immunochemically measurable amount of the specific enzyme protein (5). Being inducible by its specific substrate (6) and, through a different mechanism involving RNA synthesis (7), by glucocorticoids $(8,9)$, it has served as the model of mammalian adaptive enzymes and has drawn attention to the important physiological regulation of metabolism mediated by changes in the amounts of enzymes $(10,4)$. The quantitative study of tryptophan pyrrolase in human liver appeared to be necessary for approaching the causes that underlie the abnormal tryptophan metabolism in various diseases.

\section{Methods}

The hospitalized subjects with various diseases were all ambulatory and in no acute distress, with the indicated exception of those who were undergoing abdominal surgery. They were kept on a regular hospital diet and received no medications for at least 1 week before, and during, these studies. Unless otherwise indicated, no patient was included who had been taking corticosteroids, sedatives, tranquilizers, or vitamin preparations for a prolonged period of time. The urinary excretion of kynurenine during the 24 hours after an oral dose of $2 \mathrm{~g}$ L-tryptophan was determined by the method of Tompsett (11).

Specimens of human liver were obtained by Menghini needle biopsy by the standard percutaneous aspiration technique. When indicated, larger liver samples were obtained by excision from surgical patients during laparotomy. With these larger samples, a microassay suitable 
for the estimation of tryptophan pyrrolase in the needle biopsy samples of human liver was developed. This assay (as explained in the Results) requires fresh mitochondria that were obtained from rat liver at 0 to $4^{\circ} \mathrm{C}$ by the following procedure. One $\mathbf{g}$ of rat liver was homogenized in $7 \mathrm{ml} 0.14 \mathrm{M}$ potassium chloride and centrifuged for 10 minutes at $600 \times g$. The supernatant was centrifuged for 10 minutes at $10,000 \times g$. The sedimented mitochondria were washed once by resuspension in fresh potassium chloride solution and recentrifuged. The sediment, suspended in $35 \mathrm{ml}$ water, will be referred to as the standard mitochondrial preparation. It was used immediately.

Each needle biopsy sample of human liver (approximately 10 to $20 \mathrm{mg}$ wet weight) was immediately placed in a tissue grinder (surrounded by ice) with $0.45 \mathrm{ml}$ of $0.14 \mathrm{M}$ potassium chloride solution containing L-tryptophan $(6 \mathrm{mM})$ and sodium phosphate buffer $(\mathrm{pH} 7.2$, $0.02 \mathrm{M})$. It was homogenized by hand within 30 minutes and centrifuged in a Spinco model $\mathrm{L}$ ultracentrifuge, at $100,000 \times g$ for 45 minutes. (The above preparation of rat liver mitochondria was carried out during this time.) The assay of tryptophan pyrrolase activity involved measurement of the increase of absorbance at $360 \mathrm{~m} \mu$ (absorption peak of kynurenine) in microcuvettes $(1 \mathrm{~cm}$ light path) placed in a Beckman DU spectrophotometer equipped with thermospacers maintained at $37^{\circ} \mathrm{C}$ and a Gilford attachment for continuous recordings. Each cuvette contained $0.1 \mathrm{ml}$ of the human liver supernatant (the particle-free, soluble fraction, or "cell sap"), L-tryptophan ( $3 \mathrm{mM})$, sodium phosphate buffer $(\mathrm{pH} 7.2$, $0.02 \mathrm{M}), 0.05 \mathrm{ml}$ of the standard mitochondrial preparation, and $0.05 \mathrm{mg}$ methemoglobin, in a total volume of $0.5 \mathrm{ml}$. The principles underlying this method and some properties of the enzyme will be described in the Results.

The enzyme activity was calculated from the optical density change, which, after the lag period of 1 to $5 \mathrm{~min}$ utes, was linear for at least 30 minutes. Each assay included a control cuvette without liver supernatant that showed no significant change in optical density. The protein content of the liver supernatant was estimated by a biuret method (12). The activity of tryptophan pyrrolase was expressed as micromoles of kynurenine formed per hour per $100 \mathrm{mg}$ soluble protein. Using the soluble protein as the basis eliminates errors arising from different degrees of completeness of homogenizations.

\section{Results}

A system for the assay of rat liver tryptophan pyrrolase which ensures that the enzyme concentration alone is limiting (13) was adapted to microscale by the previously described "continuous method" (14) in which the formation of kynurenine is measured directly during incubation with tryptophan. Biopsy samples of liver obtained by surgical excision provided enough material to test the suitability of the assay system and to investigate some of the properties of human tryptophan pyrrolase. As in other mammalian livers $(3,15)$, the tryptophan pyrrolase of human liver is localized in the soluble, particulate-free phase (cell sap), and upon incubation with tryptophan, kynurenine rather than formylkynurenine accumulates owing to the high formylase activity. In normal rat liver less than one-third of the tryptophan pyrrolase is in the active holoenzyme form (15). Full activation requires the addition of its heme coenzyme in the form of hematin or methemoglobin $(15,16)$, as well as small amounts of microsomes and mitochondria (13). Figure 1 illustrates that, like rat liver cell sap, the tryptophan pyrrolase activity of human liver cell sap is doubled by the addition of methemoglobin and further increased by mitochondria. Steady rates of product formation are usually preceded by a short lag period. Throughout this study tryptophan pyrrolase activities were calculated from curves such as those in Figure 1. The cofactors contributed by rat or human liver

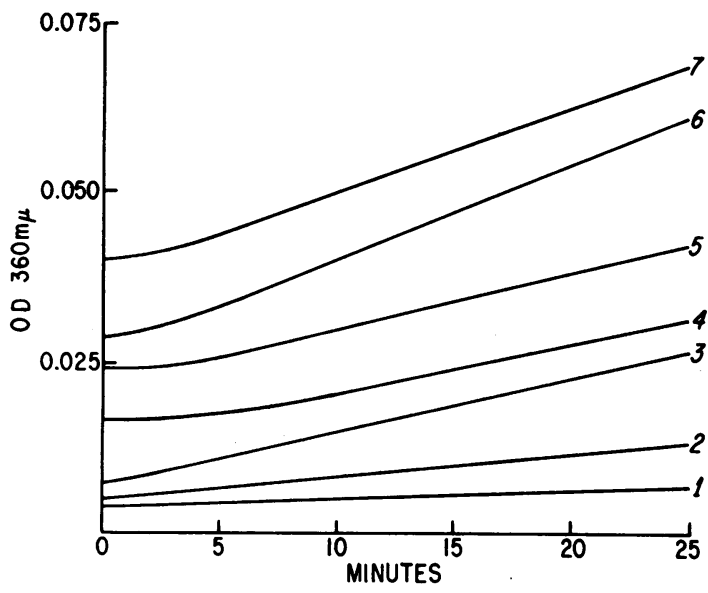

Fig. 1. IlluUstration of the Measurement of TRYPTOPHAN PYRROLASE ACTIVITY. One $g$ of surgical excision biopsy sample was homogenized in $20 \mathrm{ml}$ medium but otherwise treated as described in the Methods. Curves are reproduced from the spectrophotometric records obtained with the Gilford automatic equipment that records the optical density for each of four cuvettes, four times per minute. They were transposed arbitrarily along the ordinate to avoid superimposition of curves. The content of each of the seven cuvettes was described under Methods, with the following modifications: curve 1, no enzyme; curve 2 , no methemoglobin or mitochondria; curve 3 , no mitochondria; curves $4,5,6$, and 7 , mitochondria diluted 1 to 8,1 to 4,1 to 2 , and undiluted, respectively. 
mitochondria are equally effective in increasing the activity of tryptophan pyrrolase in human liver cell sap. Owing to the small amount of human biopsy material available, the mitochondria were routinely prepared from rat liver. They were free of tryptophan pyrrolase activity (13) as seen by the lack of optical density change in cuvettes (curve 1, Figure 1) containing all the assay constituents including mitochondria, but no soluble fraction of the liver. Such control cuvettes were run with each sample of human liver. A cuvette containing the soluble liver preparation but no mitochondria or methemoglobin (curve 2, Figure 1) was also included in each test. This was done because in rat liver after the administration of large doses of tryptophan [but not of hydrocortisone (9)] and in experimental porphyria (17) a qualitative change in the enzyme occurs: it is in the activated form and does not require the addition of methemoglobin and mitochondria. None of the enzymes of subjects in the present study showed such a change, and therefore only the activities obtained with optimal supplementation with the necessary activators will be reported here.

Table I shows that the tryptophan pyrrolase activity of the samples taken by needle biopsy is the same as the activity of the excision biopsy samples ( $1 \mathrm{~g}$ homogenized in $20 \mathrm{ml}$ medium) from the same liver. The last four subjects in Table I illustrate the reproducibility of the assay. The tryptophan pyrrolase activities of different biopsy samples of the same individual are in agreement. The activity (per $100 \mathrm{mg}$ protein) was indepen-

TABLE I

Tryptophan pyrrolase activities of different samples if human liver*

\begin{tabular}{|c|c|c|c|}
\hline \multirow[b]{2}{*}{ Subject } & \multirow[b]{2}{*}{ Assay } & \multicolumn{2}{|c|}{ Tryptophan pyrrolase activity } \\
\hline & & $\begin{array}{c}\text { Excision } \\
\text { biopsy }\end{array}$ & Needle biopsy \\
\hline & & \multicolumn{2}{|c|}{ $\mu$ moles/hour/100 mg soluble protein } \\
\hline $\begin{array}{r}4 \\
4 \\
4 \\
5 \\
7 \\
7 \\
12 \\
17\end{array}$ & $\begin{array}{l}\text { No mhb, no mt } \\
\text { No mt } \\
\text { Complete mixture } \\
\text { Complete mixture } \\
\text { Complete mixture } \\
\text { Complete mixture } \\
\text { Complete mixture }\end{array}$ & $\begin{array}{l}0.31 \\
0.64 \\
1.18\end{array}$ & $\begin{array}{l}0.39 \\
0.58 \\
1.08 \\
1.08(1.0), \dagger 1.04(1.1) \\
1.32(0.8), 1.14(1.3) \\
0.68(0.6), 0.65(1.2) \\
0.35(1.2), 0.32(0.55)\end{array}$ \\
\hline
\end{tabular}

* Needle biopsy samples (and the additional surgical excision biopsy sample from Subject 4) were assayed according to the standard procedure (complete mixture) or with the indicated omissions of methemoglobin (mhb) or mitochrondria (mt). From Subjects 5, 7, 12, and 17, needle biopsy samples were obtained and assayed on two different occasions 7 to 10 days apart. For description of subjects see Table II. The mill
parentheses. dent of the amount of protein within the range of 0.5 to $1.5 \mathrm{mg}$ per cuvette.

Table II shows the tryptophan pyrrolase levels and kynurenine excretions of a group of hospitalized subjects. Patients with rheumatoid arthritis formed one-third of the group, although we did not intend to contrast subjects with this disease with others or with the normal population; our purpose was merely to investigate a group heterogeneous with respect to the amount of urinary kynurenine after a tryptophan load. Rheumatoid arthritis is one of the conditions in which increased kynurenine excretion is frequently found (18), and this is seen in some of the subjects in the present study. Table II indicates that irrespective of the diagnosis, those subjects who excrete much kynurenine also have a high tryptophan pyrrolase activity in the liver. A further illustration of this correlation will be seen in Figure 3.

As mentioned in the introduction, the level of tryptophan pyrrolase increases in vivo upon the administration of tryptophan (3) or hydrocortisone to rats (8). Substrate induction was not attempted in humans, owing to the toxicity of the high dose (100 mg per $100 \mathrm{~g}$ body weight of rats) of tryptophan required. The smaller dose $(2 \mathrm{~g}$ tryptophan) given as a test load in the present studies did not affect the tryptophan pyrrolase level. Induction of human liver tryptophan pyrrolase by hydrocortisone, already briefly reported (19), is illustrated in Table III. Increases in the enzyme level of two-, three-, and fourfold were seen in the three subjects studied. Kynurenine excretions were measured in the same subjects. Before receiving hydrocortisone, these were in the normal range ( 30 to $80 \mu$ moles). Two to 6 days later the same patients received hydrocortisone. The tryptophan load was given $\mathbf{5}$ hours later (immediately after taking a liver biopsy sample) because, judging from observations in other species, it takes at least 5 hours for the enzyme to attain a maximal level, and then it stays elevated for another 6 to 8 hours. Most of the administered tryptophan is metabolized during this period (20). Table III shows that hydrocortisone administration raised the excretion of kynurenine in these patients to 187,135 , and $238 \mu$ moles, respectively. The values are in the range of the elevated excretions of kynurenine found in various disease states (21). The results in Tables II and 
TABLE II

Tryptophan pyrrolase level and excretion of kynurenine in hospilalized subjects

\begin{tabular}{|c|c|c|c|c|c|}
\hline Patient & Age & Sex & Diagnosis & $\begin{array}{c}\text { Tryptophan } \\
\text { pyrrolase } \\
\text { activity* }\end{array}$ & $\begin{array}{l}\text { Urinary kynurenine } \\
\text { after tryptophan load }\end{array}$ \\
\hline & years & & & $\begin{array}{l}\text { Mmoles } \\
\text { hour/ } \\
100 \mathrm{mg} \\
\text { soluble } \\
\text { protein }\end{array}$ & $\mu$ moles $/ 24$ hours \\
\hline 1 B.B. & 58 & $\mathbf{M}$ & Cancer of head of pancreas & 0.26 & $30(30) \dagger$ \\
\hline 2 C.M. & 29 & $\mathbf{M}$ & Fracture of ankle & 0.28 & $40(55)$ \\
\hline 3 G.M. & 18 & $\mathbf{M}$ & Hepatitis & 0.20 & $45(40)$ \\
\hline 4 M.F. & 45 & $\mathrm{~F}$ & Rheumatoid arthritis & 0.40 & $61(66,66,71)$ \\
\hline 5 N.F. & 22 & $\mathbf{M}$ & Erosive gastritis & 0.34 & $65(75)$ \\
\hline 6 B.C. & 23 & $\mathrm{M}$ & Fracture of humerus & 0.38 & $71(76)$ \\
\hline 7 F.H. & 38 & $\mathrm{M}$ & Cirrhosis of liver & 0.53 & $80(81)$ \\
\hline 8 R.O. & 33 & $\mathrm{M}$ & Pancreatic cyst & 0.32 & $92(81)$ \\
\hline 9 J.D. & 49 & $\mathbf{M}$ & Rheumatoid arthritis & 0.50 & $111(136)$ \\
\hline 10 B.T. & 37 & $\mathrm{~F}$ & Rheumatoid arthritis & 0.56 & $136(141)$ \\
\hline 11 L.W. & 66 & $\mathrm{~F}$ & Rheumatoid arthritis & 0.68 & $141(150)$ \\
\hline 12 L.C. & 47 & $\mathrm{M}$ & Primary amyloidosis & 0.70 & $156(175)$ \\
\hline 13 L.B. & 52 & $\mathbf{M}$ & $\begin{array}{l}\text { Malnutrition with pyridoxine } \\
\text { deficiency }\end{array}$ & 0.32 & $212(247)$ \\
\hline 14 M.K. & 59 & M & Gout, tophaceous & 1.02 & $240(232,247)$ \\
\hline 15 M.B. & 53 & $\mathrm{~F}$ & Rhuematoid arthritis & 1.20 & $262(260,265,262)$ \\
\hline 16 R.S. & 46 & $\mathrm{~F}$ & Lupus, discoid & 1.10 & $266(262)$ \\
\hline 17 T.S. & 64 & $\mathrm{M}$ & Choledocholithiasis & 1.08 & $292(272)$ \\
\hline 18 M.M. & 52 & $\mathrm{~F}$ & Rheumatoid arthritis & 1.04 & $303(313,288)$ \\
\hline 19 J.M. & 62 & M & Rheumatoid arthritis & 1.32 & $328(317,328,313)$ \\
\hline
\end{tabular}

* The tryptophan pyrrolase activity of Subjects 1,5 , and 8 was measured in surgical excision biopsy samples and of the other subjects in needle biopsy samples of liver.

$\dagger$ Repeat measurements of kynurenine of the same patient 1 to 2 weeks before biopsy are in parentheses.

III suggest that subjects with high tryptophan pyrrolase activity form more kynurenine and excrete that which accumulates because it exceeds the normal capacity of the enzyme to catabolize kynurenine.

Figure 2 illustrates the effect of pyridoxine on tryptophan pyrrolase level and kynurenine excretion. When a nutritionally normal subject who had a somewhat elevated tryptophan pyrrolase level and increased urinary kynurenine (Subject 11, Figure 2) was treated with pyridoxine, the tryptophan pyrrolase level and hence the capacity to form kynurenine remained high, but the excre- tion of kynurenine decreased. Presumably the pyridoxal phosphate enzymes that catabolize kynurenine became more active. Thus, the effectiveness of pyridoxine does not necessarily indicate that there was an "abnormal block" but, in this case, a "block" relative to increased demand. Even in healthy control subjects given a test load of tryptophan, the excretion of kynurenine was reported to decrease as a result of administration of pyridoxine (22). A patient was also studied who had chronic malnutrition with pyridoxine deficiency (Subject 13, Figure 2). As is typical for pyridoxine deficiency, this patient ex-

TABLE III

The effect of hydrocortisone on tryptophan pyrrolase and urinary kynurenine*

\begin{tabular}{|c|c|c|c|c|c|c|}
\hline \multirow{4}{*}{$\begin{array}{l}\text { Subject } \\
\text { Urinary kynurenine, } \mu \text { moles/ } \\
24 \text { hours } \\
\text { Tryptophan pyrrolase activity, } \\
\text { pmoles/hour/100 mg soluble } \\
\text { protein }\end{array}$} & \multicolumn{3}{|c|}{ Before hydrocortisone } & \multicolumn{3}{|c|}{ After hydrocortisone } \\
\hline & 4 & 3 & 2 & 4 & 3 & 2 \\
\hline & 61 & 45 & 40 & 187 & 135 & 238 \\
\hline & 0.40 & 0.20 & 0.28 & 0.85 & 0.75 & 1.18 \\
\hline
\end{tabular}

* Needle biopsy samples were obtained from Subjects 4, 3, and 2 ( $c f$. Table II). Oral tryptophan was then given and the 24-hour urine collection begun. Seven to 10 days later the same patients were injected intramuscularly with $250 \mathrm{mg}$ hydrocortisone hemisuccinate (Upjohn, Kalamazoo, Mich.). Five hours later, needle biopsy samples were taken, the tryptophan load was given, and urine collection was started. 
creted large amounts of kynurenine, which returned to normal levels after pyridoxine treatment. The purpose of illustrating these two subjects in the same Figure is to contrast the two kinds of enzymic imbalance. In the latter case the imbalance is due to abnormally low levels of the enzymes that catabolize kynurenine compared to the normal level of tryptophan pyrrolase. In the former case the enzymes that catabolize kynurenine are normal, but their activity is low compared to the abnormally high tryptophan pyrrolase activity. Pyridoxine removes the symptom, the increased excretion of kynurenine, in both cases.

Figure 3 illustrates the spectrum of tryptophan pyrrolase levels and kynurenine excretions collected in this study Only one point, that referring to the patient with pyridoxine deficiency described in Figure 2, deviates significantly from the direct correlation between these two parameters. The nutritionally normal group as a whole, including patients with rheumatoid arthritis or other conditions, exhibits a direct correlation between tryptophan pyrrolase levels and kynurenine excretion throughout the range of values. Furthermore,

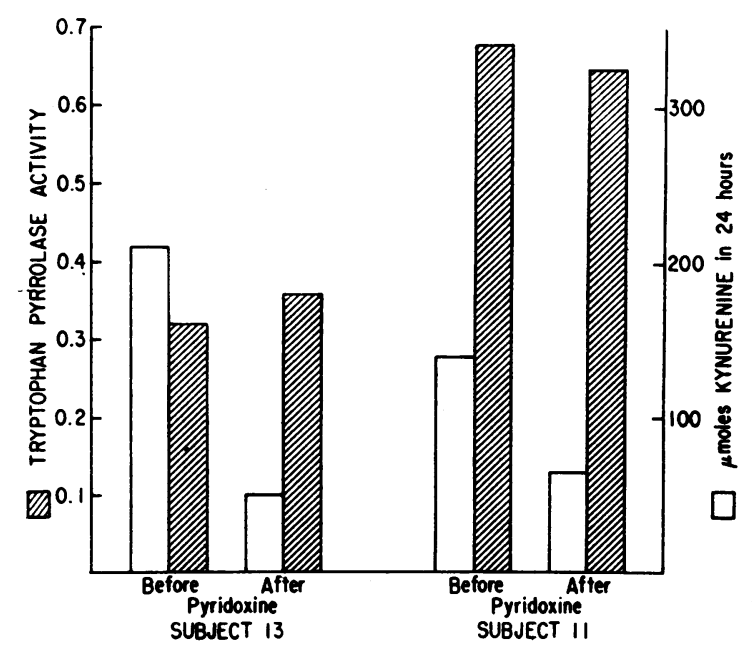

Fig. 2. The efFect OF PYRIDOXINe tREATMENT IN A PYRIDOXINE-DEFICIENT AND A NUTRITIONALLY NORMAL SUBJECT. The tryptophan pyrrolase level (dark bars, left ordinate) and, after the tryptophan load, the excretion of kynurenine (light bars, right ordinate), were determined in two subjects, one suffering from chronic malnutrition with pyridoxine deficiency (Subject 13) and another from rheumatoid arthritis (Subject 11). The same experiments were repeated on each subject after 2 weeks of daily administration of $50 \mathrm{mg}$ pyridoxineHCL.

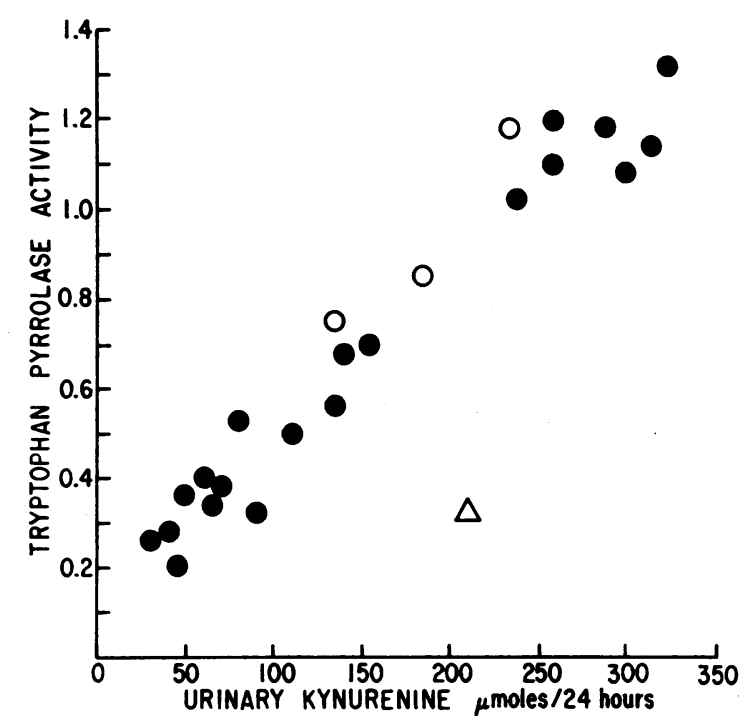

Fig. 3. ThE CORRELATION BETWEEN LIVER TRYPTOPHAN PYRROLASE ACTIVITY AND KYNURENINE EXCRETION IN HOSPITALIZED SUBJECTS. The values for tryptophan pyrrolase activity (ordinate) and urinary kynurenine per 24 hours (abscissa) are derived from Tables II and III. Circles represent nutritionally normal subjects (closed circles, untreated; open circles, after hydrocortisone). Triangle represents the untreated pyridoxine-deficient subject (see also Figure 2).

subjects whose tryptophan metabolism has been changed by hydrocortisone administration also fit this same correlation between the level of tryptophan pyrrolase and the amount of kynurenine excreted.

\section{Discussion}

The present study contributes two simple facts necessary for approaching the mechanism of increased excretion of kynurenine in subjects not suffering from pyridoxine deficiency. a) The level of tryptophan pyrrolase correlates with the amount of kynurenine excreted, and $b$ ) hydrocortisone can raise the level of liver tryptophan pyrrolase and simultaneously raise the excretion of kynurenine in man. The relevance of these two observations will be discussed separately.

a) It is reasonable to assume that the correlation between kynurenine excretion and liver tryptophan pyrrolase activity in man, shown in Figure 3 , is a causal one. There is evidence in rats that the activity of tryptophan pyrrolase measured in freshly excised livers provides a good approximation of its physiological effectiveness in situ 
and that this enzyme regulates the rate of degradation of tryptophan at low or high levels of circulating tryptophan. For example, rats whose liver tryptophan pyrrolase level has been artificially raised by the administration of the nonmetabolizable analogue, $\alpha$-methyltryptophan, oxidize more tryptophan from their body stores (23). The rate of clearance from the blood of high doses of administered tryptophan also correlates with the level of tryptophan pyrrolase as measured in liver extracts (20).

b) In intact rats, exposure to cold (24) and a variety of administered substances including aspirin $(25)$, chlorpromazine, and reserpine $(9,26)$ cause as much rise in tryptophan pyrrolase levels as do high doses of glucocorticoids. In adrenalectomized rats, only glucocorticoids and tryptophan are good inducers because the other agents act by stimulating the pituitary-adrenal system (6). As shown in the present study, the enzyme in human liver is also inducible by hydrocortisone in vivo. Thus, any form of stress or treatment with a variety of drugs that act directly or indirectly to stimulate the pituitary-adrenal system may also increase the level of tryptophan pyrrolase. The possibility that factors altering adrenocortical function underlie the increased excretion of tryptophan metabolites in a number of disparate diseases reported in the literature must be seriously considered.

The steady state concentration of kynurenine and hence its excretion can, of course, rise as a result of either a relative increase in its rate of formation or a relative decrease in its rate of degradation. In both cases, pyridoxine can lower its excretion because even in nutritionally normal subjects pyridoxal phosphate is not in excess, and by providing an excess, the appropriate enzymes can be activated to catabolize high levels of kynurenine (cf. Figure 2). Therefore, the so-called "correction" of the abnormally elevated kynurenine excretion by treatment with pyridoxine does not necessarily mean that the underlying enzymic disproportion arose from a subnormal activity of the enzymes that degrade kynurenine. This abnormality, as well as its correction by pyridoxine (which raises the activity of the degrading enzymes to above normal levels), is equally consistent with the alternative cause, an enzymic disproportion due to higher than normal activity of the first enzyme on the tryptophan-kynurenine pathway.

The excretion of metabolites of kynurenine is also governed by their steady state concentration, which depends on both the kynurenine input and the activity of the enzymes that catabolize them. Beyond a certain input of kynurenine, the capacity of some enzymes may be exceeded, and consequently the relative amounts of the various metabolites excreted will be different from those at lower inputs of kynurenine. Thus, such differences in pattern, reported to exist among different diseases (21), do not necessitate the assumption of more than one "enzymic abnormality," or in fact, any deviation from the normal activity of enzymes that catabolize kynurenine. A single cause, the (more or less) elevated level of tryptophan pyrrolase, may be responsible for the large kynurenine excretion and also for the different relative patterns of subsequent metabolites in different diseases.

When additional measurements of the relative rates of formation and removal of kynurenine become available, it will be possible to classify the diseases characterized by elevated excretions of kynurenine into at least two broad categories: 1) those in which the tryptophan pyrrolase level and the rate of production of kynurenine are normal but the degradation of kynurenine is inhibited, and 2) those in which the tryptophan pyrrolase level is increased whereas the degradation of kynurenine remains normal. Pyridoxine deficiency belongs to the first category and up to now was the only condition in which the increased excretion of kynurenine was rationally explained. There may be other diseases that exemplify the first category, but there is no clear evidence for this yet. In view of the present results, most of the conditions in which there is elevation of kynurenine excretion probably belong to the second category. Causes in addition to the hydrocortisone effect we have demonstrated can be visualized for the high tryptophan pyrrolase levels in this diverse group of diseases. The nonhormonal induction of tryptophan pyrrolase in animals is regulated by the concentration of its specific substrate and its heme coenzyme (27), and these mechanisms offer ways in which this enzyme could be elevated in appropriate disease states. For example, human cases of porphyria show increased 
excretion of tryptophan metabolites (21), and in rats with experimental porphyria the tryptophan pyrrolase level is high as a consequence of induction by the heme coenzyme that is formed in excess (17). Other possibilities are genetic variants that might cause the excessive production of this enzyme. However, increased activity (due to a stressful component of the disease or to the therapeutic measures taken) would be expected to account frequently for the observed increases in the excretion of kynurenine or its metabolites. This may represent the most common cause for the common sign of increased kynurenine excretion in a group of diseases otherwise disparate in symptomatology and origin.

\section{Summary}

An assay system suitable for measuring the activity of tryptophan pyrrolase in needle biopsy samples of human liver was developed. In a group of hospitalized subjects, the tryptophan pyrrolase activity varied between 0.26 and $1.32 \mathrm{U}$, and in the same patients, the excretion of kynurenine during 24 hours after a 2-g oral dose of tryptophan ranged between 30 and $328 \mu$ moles. The administration of hydrocortisone caused two- to fourfold increases in the level of tryptophan pyrrolase and in the amount of urinary kynurenine. In all the nutritionally normal subjects studied, including those given hydrocortisone, there was a direct correlation between the activity of tryptophan pyrrolase and the amount of urinary kynurenine. The results suggest that in a variety of diverse diseases (excluding pyridoxine deficiency) the high rate of excretion of kynurenine and its metabolites might be due to an increased rate of oxidation of tryptophan. The cause of this, the high level of liver tryptophan pyrrolase, may frequently be a result of increased adrenocortical secretion.

\section{Acknowledgments}

We are grateful to Dr. G. Acs for valuable suggestions, to Naomi Mendelsohn for expert technical assistance, and to Dr. W. McKinnon of the Department of Surgery, New York Medical College, for the excision biopsy samples.

\section{References}

1. Musajo, L., and C. A. Benassi. Aspects of disorders of the kynurenine pathway of tryptophan metabolism in man. Advance. clin. Chem. 1964, 7, 63.
2. Knox, W. E. Adaptive enzymes in the regulation of animal metabolism in Physiological Adaptation, C. L. Prosser, Ed. Washington, D. C., American Physiological Society, 1958, p. 107.

3. Knox, W. E., and A. H. Mehler. The conversion of tryptophan to kynurenine in liver. I. The coupled tryptophan peroxidase-oxidase system forming formylkynurenine. J. biol. Chem. 1950, 187, 419.

4. Knox, W. E., and O. Greengard. The regulation of some enzymes of nitrogen metabolism-an introduction to enzyme physiology in Advances in Enzyme Regulation, G. Weber, Ed. New York, Pergamon, 1964, vol. 3, p. 247.

5. Feigelson, P., and O. Greengard. Immunochemical evidence for increased titers of liver tryptophan pyrrolase during substrate and hormonal enzyme induction. J. biol. Chem. 1962, 237, 3714.

6. Knox, W. E. Two mechanisms which increase in vivo the liver tryptophan peroxidase activity: specific enzyme adaptation and stimulation of the pituitary-adrenal system. Brit. J. exp. Path. 1951, 32, 462.

7. Greengard, O., M. A. Smith, and G. Acs. Relation of cortisone and synthesis of ribonucleic acid to induced and developmental enzyme formation. J. biol. Chem. 1963, 238, 1548.

8. Knox, W. E., and V. H. Auerbach. The hormonal control of tryptophan peroxidase in the rat. J. biol. Chem. 1955, 214, 307.

9. Greengard, O., and P. Feigelson. A difference between the modes of action of substrate and hormonal inducers of rat liver tryptophan pyrrolase. Nature (Lond.) 1961, 190, 446.

10. Knox, W. E., V. H. Auerbach, and E. C. C. Lin. Enzymatic and metabolic adaptations in animals. Physiol. Rev. 1956, 36, 164.

11. Tompsett, S. L. The determination in urine of some metabolites of tryptophan-kynurenine, anthranilic acid and 3-hydroxyanthranilic acid-and reference to the presence of 0 -aminophenol in urine. Clin. chim. Acta 1959, 4, 411.

12. Layne, E. Spectrophotometric and turbidimetric methods for measuring proteins in Methods in Enzymology, S. P. Colowick and N. O. Kaplan, Eds. New York, Academic Press, 1957, vol. 3, p. 450.

13. Greengard, O., N. Mendelsohn, and G. Acs. Effect of cytoplasmic particles on tryptophan pyrrolase activity of rat liver. J. biol. Chem. 1966, 241, 304.

14. Greengard, O., and P. Feigelson. The purification and properties of liver tryptophan pyrrolase. J. biol. Chem. 1962, 237, 1903.

15. Feigelson, P., and O. Greengard. A microsomal ironporphyrin activator of rat liver tryptophan pyrrolase. J. biol. Chem. 1961, 236, 153.

16. Knox, W. E., M. M. Piras, and K. Tokuyama. Tryptophan pyrrolase of liver. I. Activation and assay in soluble extracts of rat liver. J. biol. Chem. 1966, 241, 297.

17. Feigelson, $P$., and $O$. Greengard. The activation and induction of tryptophan pyrrolase during experi- 
mental porphyria and by amino-triazole. Biochim. biophys. Acta (Amst.) 1961, 52, 509.

18. Flinn, J. H., J. M. Price, N. Yess, and R. R. Brown. The excretion of tryptophan metabolites by patients with rheumatoid arthritis. Arth. and Rheum. 1964, 7, 201.

19. Altman, K., and O. Greengard. Tryptophan pyrrolase induced in human liver by hydrocortisone: effect on excretion of kynurenine. Science 1966, 151, 332.

20. Knox, W. E. The regulation of tryptophan pyrrolase activity by tryptophan in Advances in Enzyme Regulation, G. Weber, Ed. New York, Pergamon, 1965 , vol. 4 , in press.

21. Price, J. Some effects of chelating agents on tryptophan metabolism in man. Fed. Proc. 1961, 20, 223.

22. Crepaldi, G., and A. Parpajola. Excretion of tryptophan metabolites in different forms of haemoblastosis. Clin. chim. Acta 1964, 9, 106.
23. Moran, J. F., and T. L. Sourkes. Induction of tryptophan pyrrolase by $\alpha$-methyltryptophan and its metabolic significance in vivo. J. biol. Chem. 1963, 238, 3006.

24. Maickel, R. P., and B. B. Brodie. Increase in rat liver tryptophan peroxidase as a response to pituitary-adrenal stimulation by various centrally acting drugs. Fed. Proc. 1960, 19, 267.

25. Chiancone, F. M. Enzyme activities of the tryptophan-nicotinic acid path in physiopathology. Ital. J. Biochem. 1964, 13, 1.

26. Kusch, T., and J. Heinrich. Die Aktivitätsveränderungen der Leber-Tryptophan-Pyrrolase und $\mathrm{Ty}$ rosin- $\alpha$-Ketoglutarat-Transaminase durch Chlorpromazin und Reserpin unter Berücksichtigung der Funktionen. Acta biol. med. germ. 1963, 10, 554.

27. Greengard, O., and P. Feigelson. The activation and induction of rat liver tryptophan pyrrolase in vivo by its substrate. J. biol. Chem. 1961, 236, 158.

\section{SPECIAL NOTICE TO SUBSCRIBERS}

Post Offices will no longer forward the Journal when you move.

Please notify The Journal of Clinical Investigation, Business Office, 10 Stoughton Street, Boston, Mass. 02118, at once when you have a change of address, and do not omit the Zip Code number. 